ECCOMAS

Proceedia
COMPDYN 2021

$8^{\text {th }}$ ECCOMAS Thematic Conference on Computational Methods in Structural Dynamics and Earthquake Engineering

M. Papadrakakis, M. Fragiadakis (eds.)

\title{
EFFECT OF 1D VERTICAL VARIABILITY OF SHEAR MODULUS ON THE SEISMIC RESPONSE OF SITES
}

\author{
Mounia Menoun HADJ BRAHIM ${ }^{1}$, Hamid AFRA ${ }^{2}$ \\ ${ }^{1}$ National Center of Studies and Integrated Research on Building Engineering (CNERIB) Algiers, \\ Algeria and University of Science and Technology Houari Boumediene (USTHB) Algiers, Algeria. \\ hadj.cnerib@gmail.com \\ ${ }^{2}$ National Center of Studies and Integrated Research on Building Engineering (CNERIB) Algiers, \\ Algeria and National Delegation of Major Risks, Algiers, Algeria. \\ hamid.afra@interieur.gov.dz
}

\begin{abstract}
Urban development is increased and the construction of building on poor quality soils becomes non avoidable which increases the geotechnical risk. Below these circumstances, the probabilistic approaches to study the behavior of a soil profile in a given environment have been proposed in recent years. The purpose of this research work is to study the effect of vertical variability of soil shear modulus on soil seismic response using the probabilistic approach. Two types of heterogeneity are considered: (1) heterogeneity due to the random distribution of the shear modulus in a soil profile; (2) heterogeneity of soil layer with shear modulus increasing linearly with depth. The first heterogeneous soil models are generated by Monte Carlo simulation. The spatial variation of the shear modulus is controlled by the variation coefficient parameters. The second heterogeneous soil models are assumed to have shear modulus increasing linearly with depth. These heterogeneous soils are generated and then integrated into the computer program based on the one dimensional wave propagation, resulting in seismic responses at the 'free field' soil. To demonstrate and show the importance of considering heterogeneities in the model, the results are compared with examples of deterministic analysis by means of the experimental value of shear modulus obtained from a specific site in Algeria.
\end{abstract}

Keywords: Shear Modulus, Heterogeneity, Random Distribution, Increasing Linearly, Soil Seismic Response.

ISSN:2623-3347 (C) 2021 The Authors. Published by Eccomas Proceedia.

Peer-review under responsibility of the organizing committee of COMPDYN 2021.

doi: $10.7712 / 120121.8841 .18527$ 


\section{INTRODUCTION}

In geotechnical practice, the determination of soil properties is based on tests carried out on a limited number of samples in the laboratory or on site. Soil state variables in space and time are due to complex geological conditions. Their properties change from a point to another. Due to the uncertainty associated with their inherent spatial variability, and limited available information, soil properties can be considered as random variables. Moreover, poor soil sampling procedures, test procedures, characteristics of the test setup device and operator experience can have a large impact on the measured geotechnical properties. The probabilistic approach is often used in engineering sciences to represent and decrease these uncertainties. It attempts to take into account all the uncertainties affecting the properties of soils, and allow the engineer to make the decision that bringing more objectivity; it assumes that the characteristics are variables defined by a mean, a standard deviation and a distribution law. To consider these uncertainties using the probabilistic approach, soil properties are treated as random variables. These random variables are functions defined on a sample space to represent quantities that can change. In practice, a random variable is where the estimated value is uncertain, but a certain probability can be assigned to any specific value (take values from a finite or countable set) "for discrete random variables" or within any value from an interval on a real line "continuous random variables".

Several studies have been conducted taking into account uncertainty in geotechnical engineering, Tian and Jie [1] conducted a study of the uncertainty of soil parameters, regarding the shear wave velocity (VS), thickness (h), density and damping for the propagating of onedimensional wave. Specific conclusions were drawn. Nour [2] studied, two-dimensional seismic response, using Monte Carlo simulations combined with the deterministic method of shear modulus, critical damping and Poisson's ratio of soil properties. The results showed a significant attenuation of the tendency of the acceleration at the ground surface compared to the homogeneous case. Dominic et al [3] carried out a study of the importance of the spatial variability of soil properties on the surface response spectrum using Monte Carlo simulation based on the Popescu methodology [4]. They concluded that local conditions are important according to the gradient of the stiffness of the profile and intensity of the movement and that further studies should be performed. Paolo and Cornell [5] presented a probabilistic work to study the effect of soil layers on the estimation of the nonlinear amplification function, AF (f) and the acceleration response spectrum for a frequency range of the oscillator of the structures. The results revealed that AF (f) depends strongly on the intensity of the input soil movement at the same oscillator frequency, for the two soil types studied (sand and clay). Andrade and Borja [6] performed a combined stochastic-deterministic analysis of the local site response. To compare the sensitivities of equivalent linear and non-linear analysis procedures, two computer codes SHAKE and SPECTRA were used. The soil variability as well as the frequency and duration of earthquakes were considered in this study. The results showed that SPECTRA has approximately the same sensitivity as SHAKE.

Other recent typical examples include the work of Li and Assimaki [7], Johari A. [8] and Menzer Pehlivan et al [9] examined the special variability of soil on the prediction of a strong soil motion, where the consequences of spatial variability on the seismic response are not well understood. The basic characteristics of any layer are the shear wave velocity, the thickness and the non-linear properties of soil. The values of these properties in nature through a site vary from one point to another and there is always uncertainty in their measurement, this is a major contributor to uncertainty in geotechnical engineering analyzes. 


\section{PROBABILISTIC METHOD OF INDIRECT SIMULATION OF NON-GAUSSIAN RANDOM FIELDS}

The probabilistic method of indirect simulation of non-Gaussian random fields is based on the theory of random fields. This approach is based on the decomposition of random variables as described by Fenton [10].

The algorithm that allows the generation of $\Delta f_{p}(z)$ is described as follows:

$$
\Delta f_{p}(z)=\sum_{n=1}^{N_{p}} \Delta g_{p n}(z)
$$

With $N p$ is the number of soil properties to be simulated. The Gaussian random field with $N p$ variables, one-dimensional, with zero mean and unity variance $\Delta g_{p n}(z)$ can be simulated as follows $[11,16]$ :

$$
\Delta g_{p n}(z)=\sqrt{2} \sum_{l=0}^{N} A_{l, p n} \cos \left(k_{l} z+\Omega_{l, p n}\right)
$$

With

For multivariate fields

$$
A_{l, p n}=H_{p n}\left(k_{l}\right) \sqrt{2 \Delta k}
$$

For uni-variate fields

$$
A_{l}=\sqrt{2 S\left(k_{l}\right) \Delta k}
$$

Equation (3) also can ensures the inter-property correlation, $\Omega_{l, p n}$ are random phases uniformly distributed in the interval $\left[\begin{array}{ll}0 & 2 \pi\end{array}\right]$. The coefficients Hpn are obtained from the inter spectral density matrix in the wave number domain $S(\mathcal{K})$, the latter is a real, symmetrical matrix, and positive definite. The elements of the matrix $H$ are obtained from the Cholesky decomposition of the symmetric matrix $\mathrm{S}$ which is defined by:

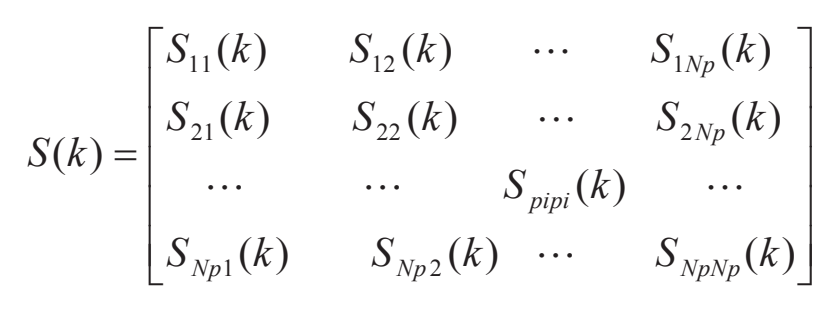

The matrix Hpn is assumed to be lower triangular:

$$
H(k)=\left[\begin{array}{cccc}
H_{11}(k) & 0 & \ldots & 0 \\
H_{21}(k) & H_{22}(k) & \ldots & 0 \\
\ldots & \ldots & \ldots & 0 \\
H_{N p 1}(k) & H_{N p 2}(k) & \ldots & H_{N p N p}(k)
\end{array}\right]
$$

with $\mathcal{K}$ is the number of waves which satisfies:

$$
\left\{\begin{array}{l}
k_{l}=l . \Delta k \\
k_{u}=N . \Delta k
\end{array}\right.
$$


The wavenumber step $\Delta \mathcal{K}$ is evaluated from the representation of $S \mathcal{K}$ and by evaluating the cut-off wavenumbers values $\mathcal{K} u$ for $N$ increments, $\mathcal{K} u$ is defined by:

$$
k_{u}=\frac{1}{a} \sqrt{\ln \left(\frac{1}{\lambda^{4}}\right)} ; \lambda=0.001
$$
data.

a is a parameter statistic, which is obtained from a non-linear regression of experimental

In this study, we consider one-dimensional, univariate, homogeneous but non-Gaussian field with mean zero and spectral density function given by [2]:

$$
S(k)=\frac{\left.\sigma^{2}\right|_{\sigma=1} a}{2 \sqrt{\pi}} \exp \left[-\left(\frac{k a}{2}\right)^{2}\right]
$$

In order to considerably reduce the computing time, the digital generation of sample functions of equation (2) is easily calculated using the Fast Fourier Transform (FFT), developed by Yamazaki and Shinozuka [17] and modified by Zerva [18].

The analysis carried out in this paper considers $\lambda$ (is a small real number close to zero) equal to 0.001 .

\section{SPATIAL VARIABILITY OF UNBOUNDED SOIL PROPERTIES}

Soil properties such as elastic modulus E, shear modulus G, permeability k, etc., are assumed to be lognormally distributed. This choice is motivated by the fact that these properties are positive parameters, and that the lognormal distribution makes it possible to analyze their great variability. The expression of the shear modulus for example is given by $[2,10]$ :

$$
\lambda_{G}(z)=\exp \left[\lambda_{0 G}(z)+\xi_{G} \Delta f_{G}(z)\right]
$$

With:

$$
\left\{\begin{array}{l}
\xi_{G}^{2}=\operatorname{Ln}\left(1+\frac{\sigma_{G}^{2}}{\lambda_{0, G}^{2}}\right) \\
\lambda_{0 G}(z)=\ln \left(\lambda_{0, G}\right)-\frac{1}{2} \xi_{G}^{2}
\end{array}\right.
$$

Where $\lambda_{0, G}$ and $\sigma_{G}^{2}$ are respectively stand for shear modulus means and variance.

$\mathrm{CV}_{\mathrm{G}}$ : Coefficient of variation: To provide a dimensionless expression of the uncertainty inherent in a random variable.

\section{HETEROGENEITY OF SOIL LAYER WITH SHEAR MODULUS INCREASING LINEARLY WITH DEPTH}

In this proposed approach the soil profile is assumed to have shear modulus increasing linearly with depth. In the upper layer, of thickness $h_{1}$, the shear modulus is supposed to vary according to [19]:

$$
G_{1}(z)=G_{0}\left(z / h_{1}\right)^{p}
$$

Where $\mathrm{z}$ is the depth counted positively downwards from ground surface, $\mathrm{G}_{0}$ is the shear modulus at depth $\mathrm{h}_{1}, \mathrm{p}$ is an arbitrary exponent, which takes values in range $0 \leq p<2$. The 
parameter $\mathrm{p}$ can be determined by a least square fit through the measured values of the shear wave velocity $\hat{V}(z)$ and is given by Ref. [20]:

$$
p=\ln V_{s 1} \frac{1}{h 1} \int_{0}^{h_{1}} \ln \left(\frac{z}{h_{1}}\right) \ln \hat{V}(z) d z
$$

Where $\mathrm{V}_{\mathrm{s} 1}$ is the shear wave velocity at depth $\mathrm{h}_{1}$

For the underlying layer the shear modulus is assumed to be:

$$
G_{2}(z)=G_{0}+G 1\left(z / h_{2}\right)
$$

Where $\mathrm{z}$ is counted from the layers interface and $\mathrm{h}_{2}$ is the thickness of second layer and $\mathrm{G}_{0}+\mathrm{G}_{1}$ is the shear modulus at depth $\mathrm{h}_{2}$.

\section{RESULTS OF THE DYNAMIC RESPONSE OF A HETEROGENOUS ONE- DIMENSIONAL SOIL PROFILE}

\section{- Site variability Effects (shear modulus): the case of Souidania}

This example deals with the vulnerability of a site with an inclined topography, with a slope that varies from $4 \%$ to $5 \%$, (figure 10). The site is located at Souidania, located in the southwestern suburbs of Algiers and occupies an area of around 6 ha [21].

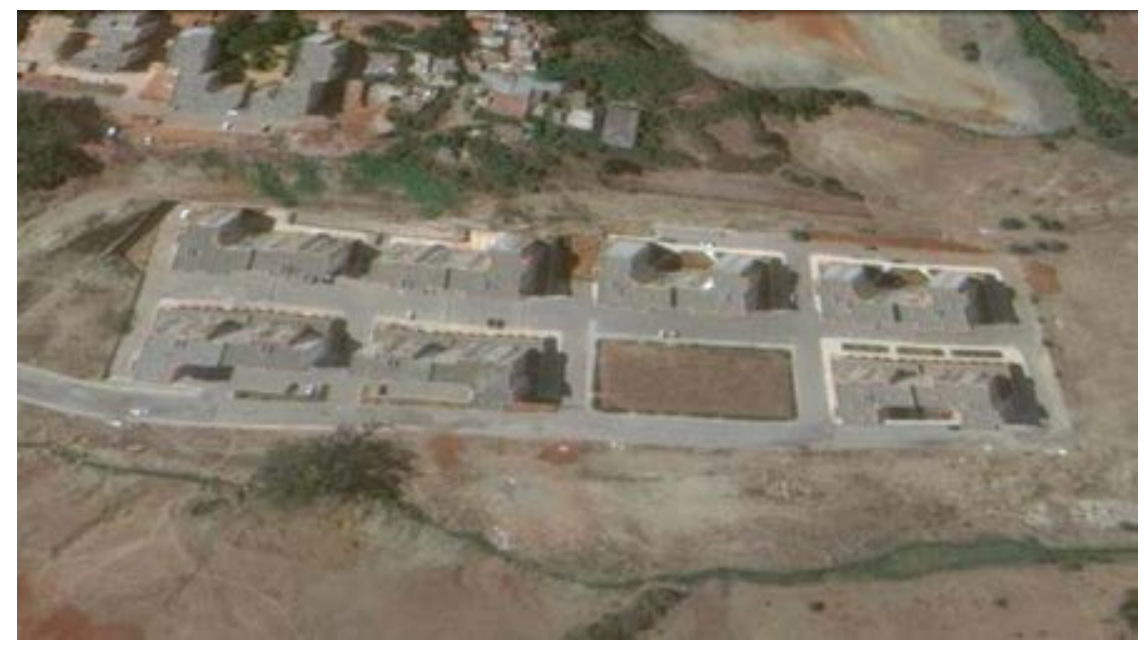

Fig. 1. Location of the project (Google Earth, October 2016)

It is shown in figures 2 and 3 ten realizations of the shear modulus for different values of $C V G$. The effect of site variability is therefore an essential parameter in an urban environment which must be evaluated to better define the lateral variability of the seismic movement of the soil, with immediate consequences on the response of structures and their damage. 
- First layer
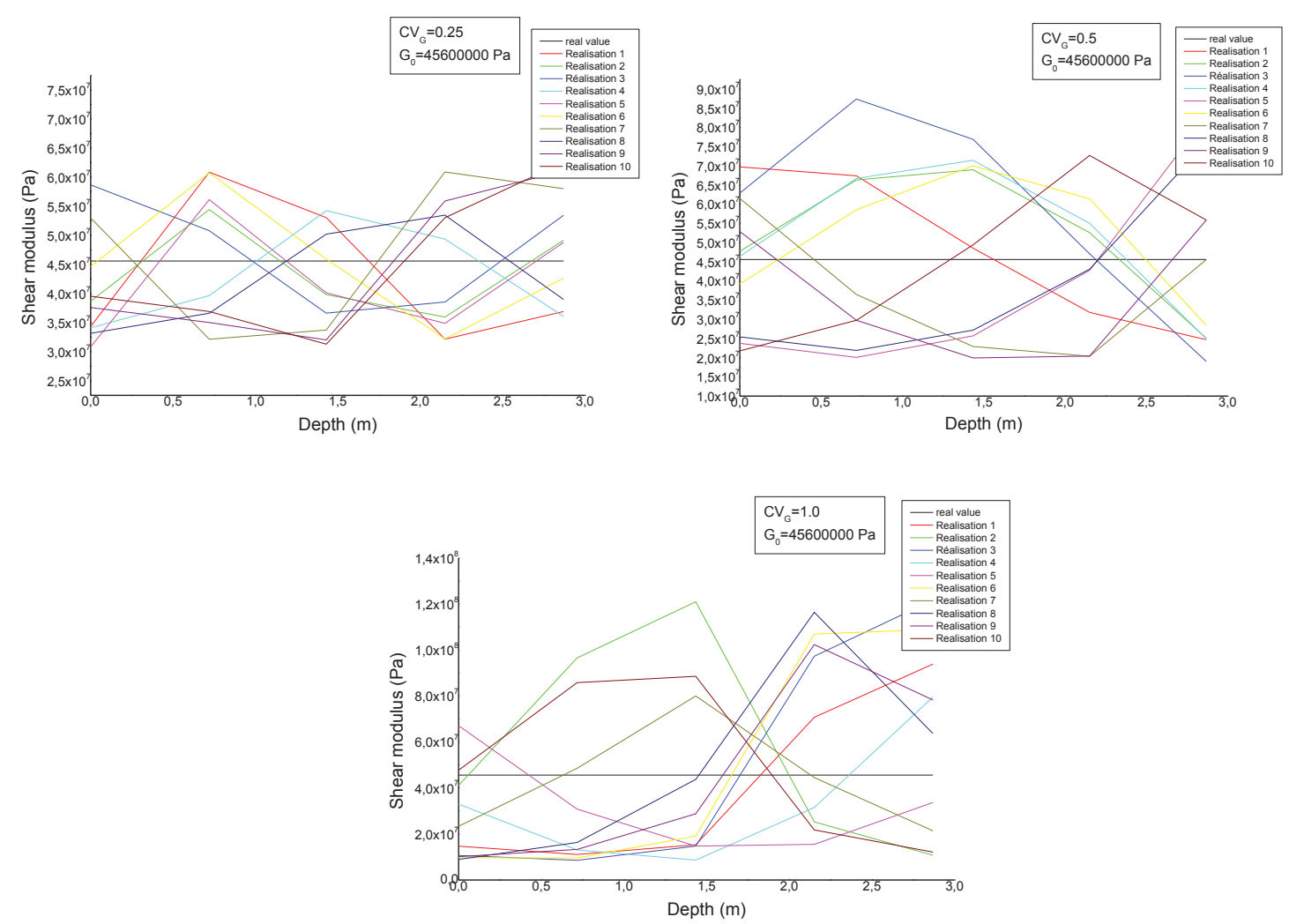

Fig. 2. Ten realizations of variations in shear modulus, first site layer, for different values of $C V_{G}$.

- Second layer
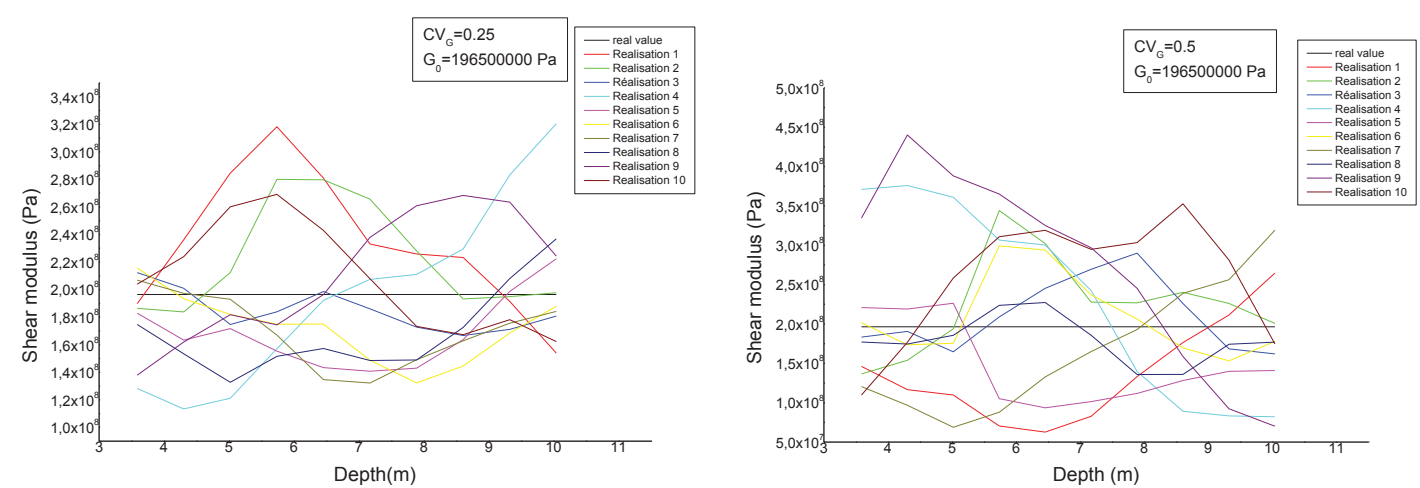


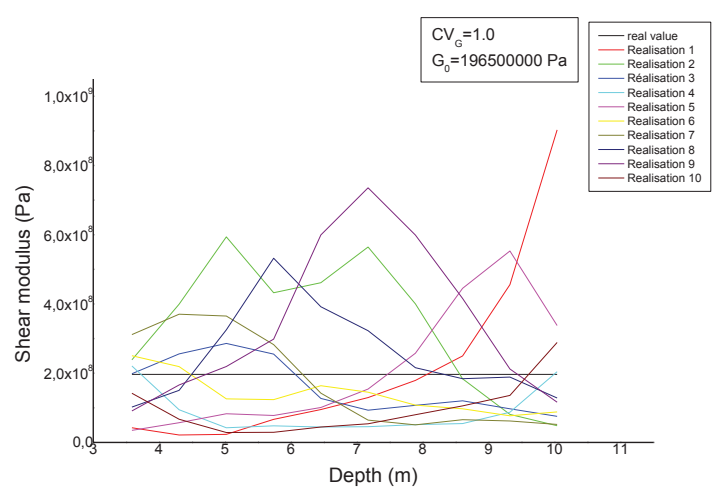

Fig. 3. Ten realizations of shear modulus variations, second site layer, for different values of $C V_{G}$.
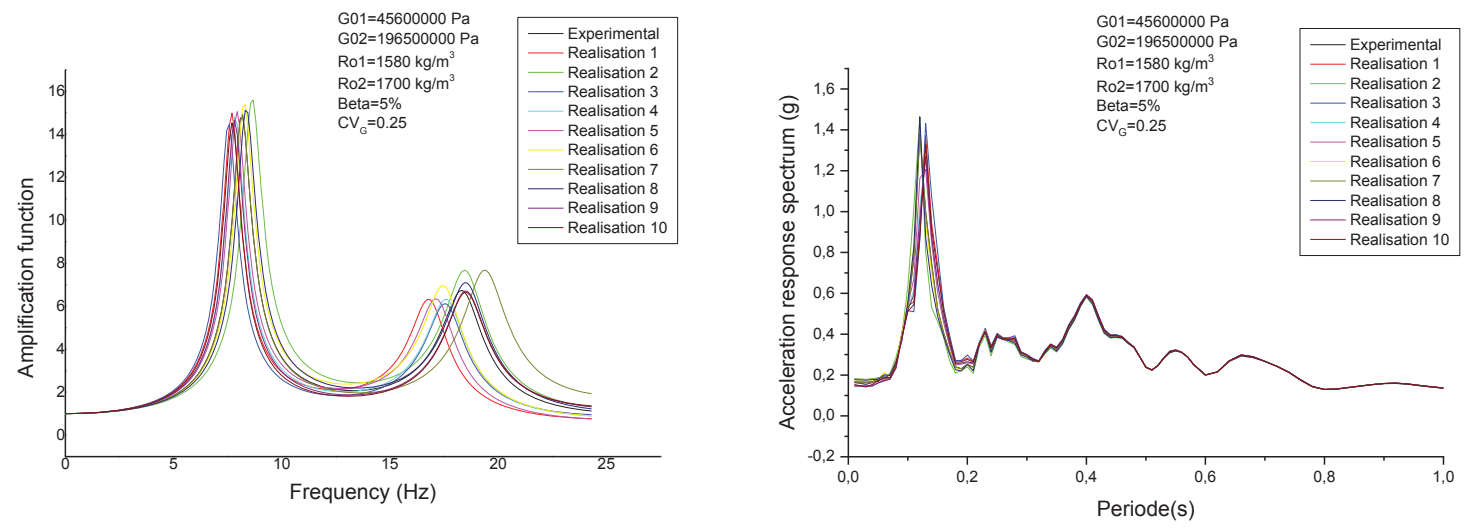

Fig. 4. Seismic amplification and the response spectrum in acceleration at the surface of the experimental case and of ten realizations according to the variability of the shear modulus $G$ for a value of $C_{V_{G}}=0.25$.
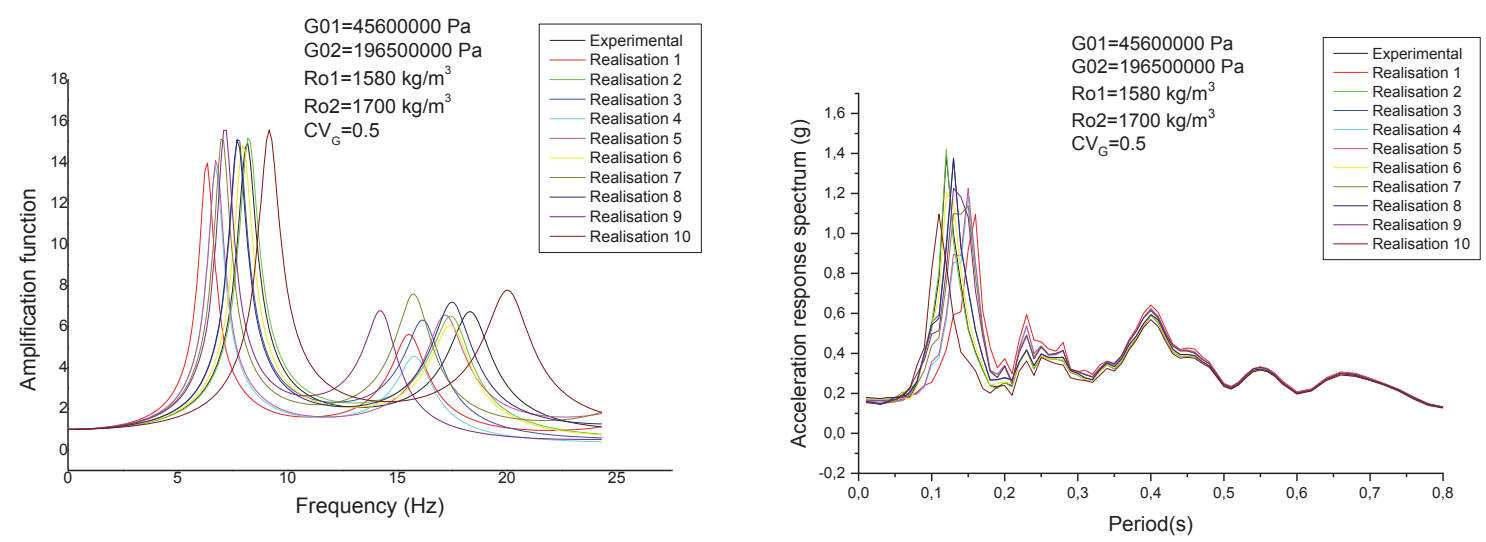

Fig. 5. Seismic amplification and the response spectrum in surface acceleration of the experimental case and ten realizations according to the variability of the shear modulus $G$ for a value of $\mathrm{CV}_{\mathrm{G}}=\mathbf{0 . 5}$. 

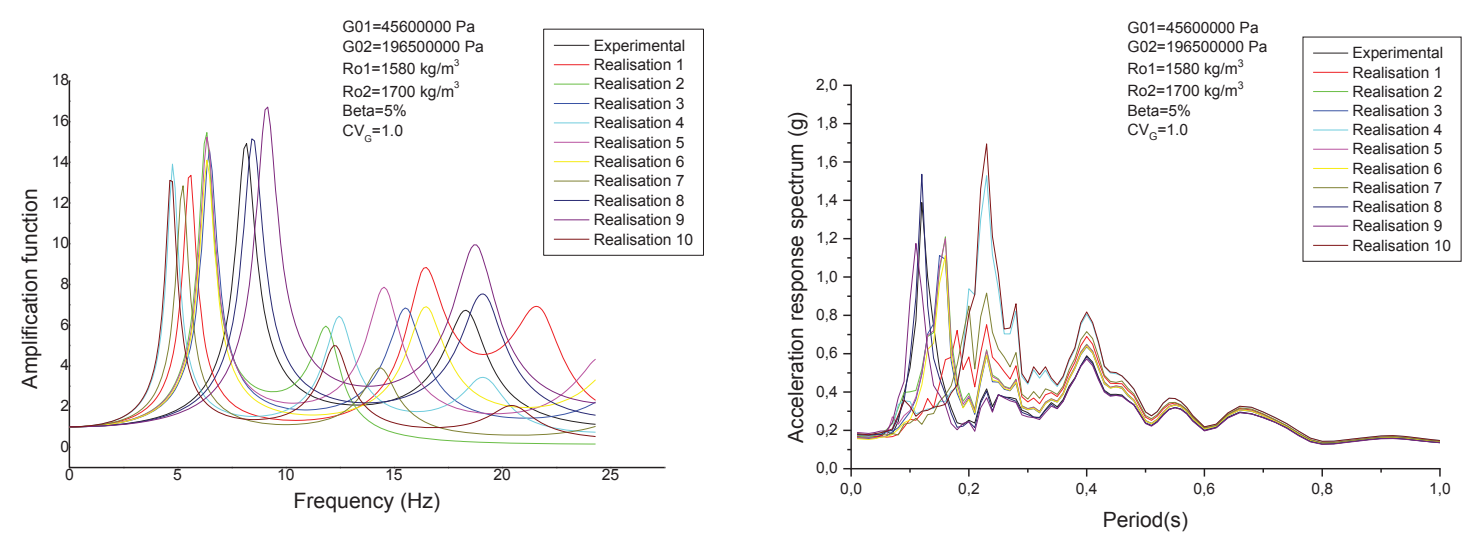

Fig. 6. Seismic amplification and the response spectrum in acceleration at the surface of the experimental case and of ten realizations according to the variability of the shear modulus $G$ for a value of $C V_{G}=1$

From figures 4, 5 and 6, it is clearly seen that the variation of the shear modulus has great importance on the seismic amplification of the soil and this amplification varies according to the coefficient of variation $\mathrm{CV}_{\mathrm{G}}$ where the value of the $\mathrm{CV}_{\mathrm{G}}$ varies from 0.25 slightly heterogeneous to the value 1.00 strongly heterogeneous. Figure 7 shows a comparison between the case of heterogeneous experimental case, the cases of average of the ten realizations for each $\mathrm{CV}$ value and the case of shear modulus increasing linearly with depth.

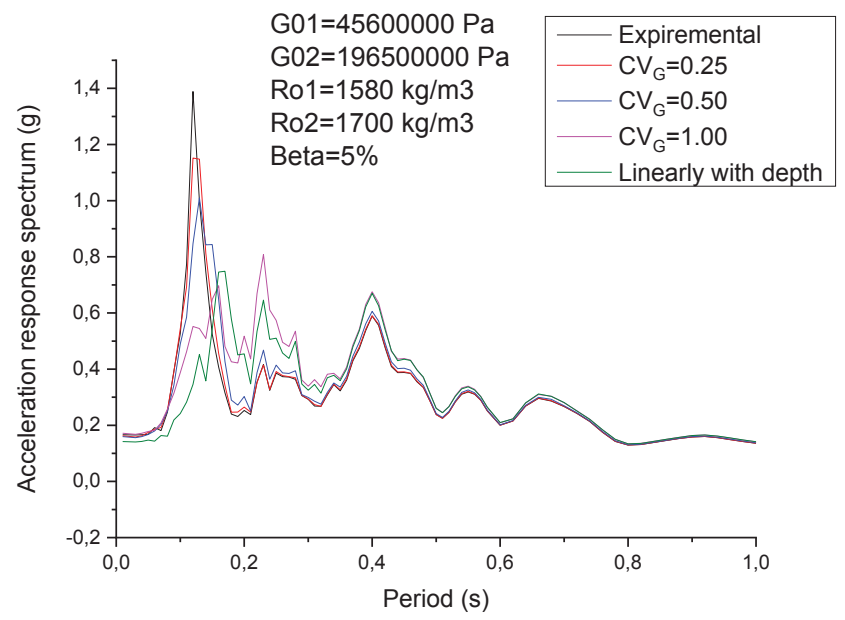

Fig.7. Surface acceleration response spectrum difference between the experimental case, the case of Monte Carlo as a function of the variability of the shear modulus $G$ for different $\mathbf{C V}_{\mathbf{G}}$ values and the case of shear modulus increasing linearly with depth.

From the curves we can clearly see the differences between the studied cases, and that the probabilistic seismic response of heterogeneous soil profile via Monte Carlo simulations, for the value of $\mathrm{CV}=0.25$, is more equivalent to that of the seismic response of experimental case. On the other hand, we have observed an attenuation compared to the other cases of the value of $\mathrm{CV}=0.5$ and $\mathrm{CV}=1$. Furthermore, For the case of the shear modulus increasing linearly with depth, the value of the response spectrum is identical to the probabilistic seismic response of heterogeneous soil profile via Monte Carlo simulations for the case of CV $=1$. 


\section{DISCUSSION AND CONCLUSIONS}

In this paper the acceleration response spectrum of the heterogeneous seismic amplification soils is analyzed. The random soil properties considered is the shear modulus, modeled by random spatial fields. The random field for the shear modulus is obtained by adopting the lognormal distribution, which allows its great variability to be analyzed.

As a summary of this article, we can recapitulate the following comments

- The increase in the coefficient of variation of the shear modulus induces a decrease in soil amplification and the peak acceleration response spectrum for the one-dimensional case. On the other hand, it is observed that increasing the coefficient of variation of the shear modulus also causes a filtering effect which slides the fundamental frequency of the system towards low frequencies, and causes attenuation of high frequencies. In this case, the simulated soil becomes softer compared to the homogeneous soil $(C V G=0)$.

- For the case of shear modulus increasing linearly with depth, the value of the response spectrum is identical to the probabilistic seismic response of heterogeneous soil profile via Monte Carlo simulations for case of $\mathrm{CV}=1$.

The results obtained indicate that the seismic response is related to the shear modulus variability. In addition, it is observed that the increase in the coefficient of variation of the shear modulus induces a decrease in the peak value of the response spectrum in acceleration for the one-dimensional case. The method described in this paper, by analyzing several realizations, can reduce the geotechnical risk.

\section{REFERENCES}

[1] Tian, L., and L. Jie. 1992. Influence of random mechanical parameter on earthquake response analysis of site, 10th World Conf. on Earthquake Engineering, Madrid, Spain, 19-24 July.

[2] Nour, A. 2004. Investigation stochastique sur les milieux hétérogènes par la méthode des éléments finis. Thèse de Doctorat, Université des Sciences et de la Technologie Houari Boumedienne, Algérie.

[3] Dominic Assimaki, Alain Pecker, Radu Popescu And Jean Prevost. 2003. Effects of spatial variability of soil properties on surface ground motion. Journal of Earthquake EngineeringVol. 07, No. spec01, pp. 1-44.

[4] Popescu, R., Prevost, J. H. and Vanmarcke, E. H. 1995. Numerical simulations of soil liquefaction using stochastic input parameters, Proc. 3rd International Conference on Recent Advances in Gmtechnical Earthquake Engineering and Soil Dynamics, St. Louis, MO.

[5] Paolo Bazzurro and C. Allin Cornell. 2004. Nonlinear Soil-Site Effects in Probabilistic Seismic-Hazard Analysis Bulletin of the Seismological Society of America, Vol. 94, No. 6, pp. 2110-2123.

[6] Andrade J.E. et Borja R.I. 2006. Quantifying sensitivity of local site response models to statisticalvariations in soil properties. Acta Geotechnica 1:3-14.

[7] Li W. and Assimaki D. 2010. Site- and Motion-Dependent Parametric Uncertainty of Site-Response Analyses in Earthquake Simulations. Bulletin of the Seismological Society of America, Vol. 100, No. 3, pp. 954-968. 
[8] Johari A. Momeni M. 2015. Stochastic analysis of ground response using non-recursive algorithm. Soil dynamics and earthquake engineering 69: 57-82.

[9] Menzer Pehlivan, Ellen M. Rathje and Robert B. Gilbert. 2016. Factors influencing soil surface seismic hazard curves. Soil dynamics and earthquake engineering 83, 180-190.

[10] Fenton G.A. 1990. Simulation and Analysis of Random Fields. Ph.D. thesis, Princeton University.

[11] Mantoglou A. and Wilson J.L. 1981. Simulation of Random Fields with the Turning Bands Method. Department of Civil Engineering, Report No. 264, Massachusetts Institute of Technology, Cambridge, MA, July.

[12] Mignolet M.P. and Spanos P.D. 1992. Simulation of homogeneous two-dimensional random fields: Part I - AR and ARMA Models. ASME J. Appl. Mech., 59: 260-269.

[13] Matheron G. 1973. The Intrinsic Random Functions and Their Applications. Advances in Applied Probability; 5: 439-468.

[14] Fenton G.A. and Vanmarcke E.H. 1991. Spatial Variation in Liquefaction Risk Assessment. Proc. Of Geotechnical Engrg. Congress, ASCE, Geotech. Div., Boulder, Colorado,594 - 607.

[15] Fenton G.A. 1994. Error evaluation of three random field generators. Journal of Engineering Mechanics ASCE, 120(12): 2478-2497.

[16] Ghanen R.G. and Spanos P.D.1991. Stochastic finite elements: a spectral approach. Springer-Verlag.

[17] Yamazaki F, Shinozuka M. 1988. Digital generation of non-Gaussian stochastic field. Journal of Engineering Mechanics ASCE; 114(7): 1183-1197.

[18] Zerva A. 1992. Seismic ground motion simulations from a class of spatial variability models. Earthquake Engineering and Structural Dynamics; 21: 351-361.

[19] Afra, H., and Pecker A. 2002. Calculation of free response spectrum of a nonhomogeneous soil deposit from bed rock response spectrum. Soil Dynamics and Earthquake Engineering, 22: 157-165.

[20] Pecker A. and Afra H. 1995. Charts for natural frequencies and transfer function on an inhomogeneous soil deposits. Fifth International Conference on Seismic Zonation, II : p. 1091-8.

[21] Rapport géotechnique sur site construction logements AADL/SOUIDANIA wilaya d'Alger 2002. Bureau d'études B.Heba. 